\title{
3D myocardial perfusion-CMR using a multi- transmit coil and k-t PCA reconstruction to detect flow limiting coronary stenosis
}

\author{
Roy Jogiya*, Amedeo Chiribiri, Andreas Schuster, Christian Jansen, Kalpa De Silva, Divaka Perera, Simon Redwood, \\ Eike Nagel, Sebastian Kozerke, Sven Plein
}

From 2011 SCMR/Euro CMR Joint Scientific Sessions

Nice, France. 3-6 February 2011

\section{Purpose}

To explore the clinical feasibility of 3D $k-t$ PCA myocardial perfusion CMR on a multi-transmit $3 \mathrm{~T}$ system in patients listed for invasive intracoronary pressure wire assessment.

\section{Background}

Three-dimensional acquisition methods facilitated by undersampling in space and/or time have been proposed to overcome the limited cardiac coverage of dynamic first pass myocardial perfusion-CMR. Recently, temporally constrained $\mathrm{k}-\mathrm{t}$ BLAST reconstruction using principal component analysis ( $k-t$ PCA) has been proposed to further improve temporal resolution of $\mathrm{k}-\mathrm{t}$ undersampled data. In addition, multi-transmit technology has become available, which aims to improve image uniformity and SAR limitations at higher field strength. We have developed a pulse sequence for 3D perfusion-CMR that utilises both k-t PCA and multi-transmit technology at 3 Tesla.

\section{Methods}

8 patients with suspected coronary artery disease underwent 3D perfusion MR imaging at rest and adenosine stress prior to angiography and fractional flow reserve assessment. $k-t$ PCA accelerated perfusion CMR was performed on a 3T Philips Achieva Multi-transmit system (3D dynamic spoiled gradient echo, TR $2.0 \mathrm{~ms}$, TE $1.0 \mathrm{~ms}$, flip angle 30 , matrix 160x160x80 mm, 16 slices of $5 \mathrm{~mm}$ thickness). FFR was measured in all vessels with visually significant stenosis using a pressure sensortipped wire $\left(\right.$ Volcano $\left.^{\circ}\right)$. FFR $<0.75$ was considered

Kings College London, London, UK

(c) 2011 Jogiya et al; licensee BioMed Central Ltd. This is an open access article distributed under the terms of the Creative Commons Attribution License (http://creativecommons.org/licenses/by/2.0), which permits unrestricted use, distribution, and reproduction in any medium, provided the original work is properly cited. haemodynamically significant. Two experienced obserinterpreted ischemia on CMR data as relative underperfusion of a sector within a slice or relative endocardial vs epicardial underperfusion within a coronary teritory. The performance of visual analysis of CMR to detect determined.

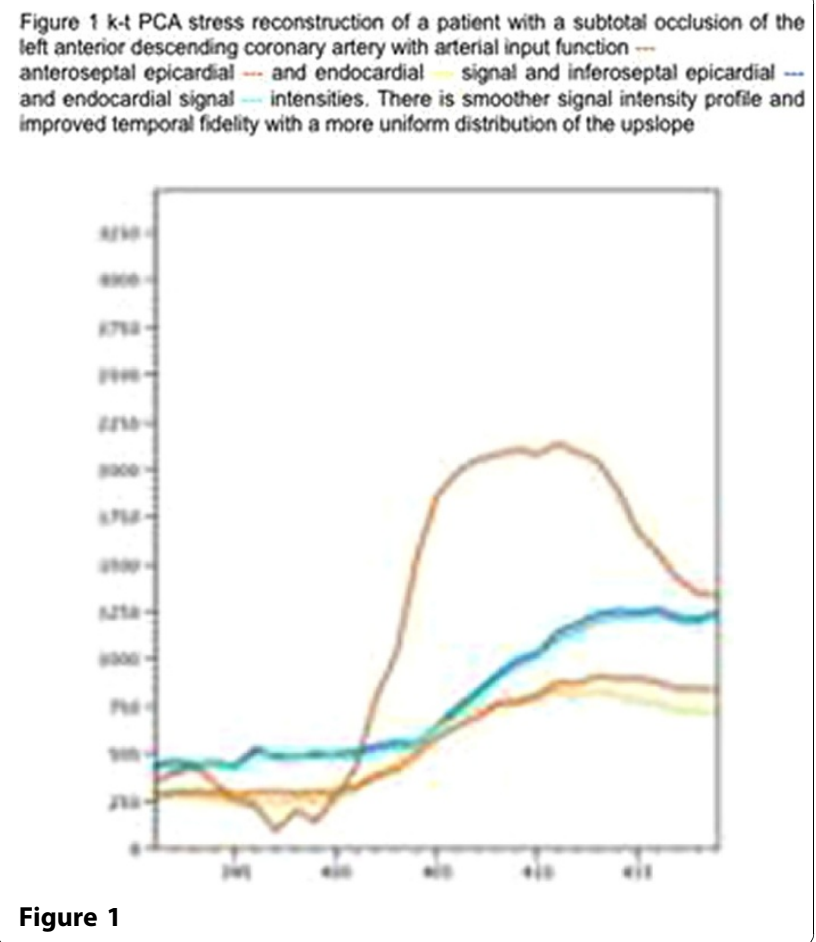
vers blinded to the results of the angiogram visually flow-limiting coronary stenosis on angiography was 


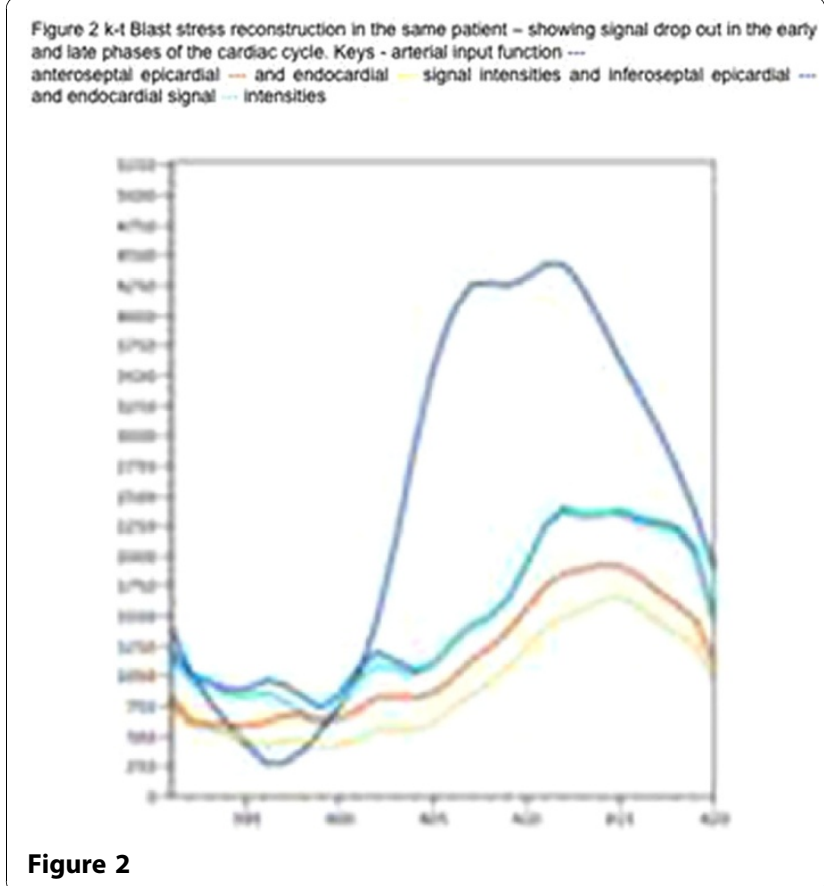

\section{Results}

Acquisition was feasible in all patients. Signal intensity (SI) profiles showed improved temporal fidelity of $\mathrm{k}-\mathrm{t}$ PCA (Figure 1) compared with standard k-t BLAST (figure 2) reconstruction SI profiles were similar across myocardial segments. Using k-t PCA Image quality was scored as "good" in 6 of the 8 cases. Perfusion deficits were seen in 4 of the 8 scans. Abnormal FFR was found in 4 patients with correlation between CMR and FFR in 6 of the 8 cases.

\section{Conclusion}

3D myocardial perfusion imaging using Multi-transmit technology and k-t PCA reconstruction is technically feasible. Improved temporal fidelity was observed using multi-transmit technology. Perfusion deficits in patients with suspected CAD correlate well with invasive FFR measurements in this small pilot study.

Published: 2 February 2011

doi:10.1186/1532-429X-13-S1-P12

Cite this article as: Jogiya et al:: 3D myocardial perfusion-CMR using a multi-transmit coil and k-t PCA reconstruction to detect flow limiting coronary stenosis. Journal of Cardiovascular Magnetic Resonance 201113 (Suppl 1):P12.
Submit your next manuscript to BioMed Central and take full advantage of:

- Convenient online submission

- Thorough peer review

- No space constraints or color figure charges

- Immediate publication on acceptance

- Inclusion in PubMed, CAS, Scopus and Google Scholar

- Research which is freely available for redistribution

Submit your manuscript at www.biomedcentral.com/submit 